\title{
Biochemical Studies on Carob
}

\author{
Nadia Y.A. Attia. ${ }^{1}$; Ibrahim M. Abd El - Aleem ${ }^{1}$; Kareem M.K. El Tobgy ${ }^{2}$ and Hala F. Mohamed ${ }^{2}$
}

1: Department of Biochemistry, Faculty of Agriculture, Moshtohor, Banha University.

2: Department of Medicinal and Aromatic Plants Research, Horticulture Research Institute, Agriculture Research Center.

Water, methanol and ethanol were used to extract total phenols, total tannins, and flavonoids from carob (Ceratonia siliqua L) by using different methods. The obtained extracts were used to study their antioxidant activity and their antimicrobial effect against some Gram positive bacteria ( Staphylococcus aureus, Listeria monocytogenese, Bacillus megaterium and Bacillus cereus), some Gram negative bacteria (Escherichia coli, Klebsiella pneumonia and Salmonella typi) and some fungi (Aspergillus niger and Candida albicans). Also, carob fiber and water extract were used to study their biological effects against diabetic and hypercholesterolemia in Wister rats. Water extract at $50^{\circ} \mathrm{C}$ for $20 \mathrm{~min}$ showed the highest capacity for extracting total phenols, total tannins and total flavonoids compounds from carob. Methanol extract at $50^{\circ} \mathrm{C}$ for 20 min and at $25^{\circ} \mathrm{C}$ for $24 \mathrm{~h}$ recorded the highest antioxidant activity. Some carob extracts recorded inhibition for the tested microorganisms except salmonella typhi which did not affected. Administration of carob fiber and water extract showed significant decrement in blood serum glucose, triglycerides, total cholesterol, low density lipoprotein (LDL), urea and creatinine in diabetic and hypercholesterolemia rats while, high density lipoprotein (HDL) elevated. Administration of carob fiber and water extract induced significant increment in white blood cells (WBC) in diabetic groups. While, hypercholesterolemia group administrated with carob fiber showed significant increment in Red blood cells (RBC) count and carob water extract showed increment in RBC count and decrement in WBC count.

Key words: Carob - antioxidant - antimicrobial - lipid profile - liver functions.

\section{Introduction}

Several years scientists are interested in studying the effect reactive oxygen species (ROS) that are implicated in many human diseases (Lobo et al., 2010). Increased ROS led to oxidative stress and a degenerative signaling cascade triggered by oxidation of vital cellular components, which induced cellular damage and cell death (Farrugia and Balzan, 2012). The carob tree has been widely cultivated for years in Mediterranean countries. Various chemical and physiological aspects of carob plants have been investigated. Hussein et al. (2011) found that the total protein, fiber, total carbohydrate, fat, ash and moisture contents in dried carob were $8.95 \%, 8.91 \%, 73.14 \%, 5.48 \%, 3.52 \%$ and $10.1 \%$ respectively. Carob extracts have several beneficial effects on health such as cholesterol lowering activities in humans suffering from hypercholesterolemia (Zunft et al., 2001, 2003) and antioxidant properties in vitro test systems (Custodio et al., 2005). Recent studies discovered that Tunisian leaf carob extract presented some ameliorative effects against $\mathrm{CCl} 4$-induced oxidative damage in rats tissues (Hsouna et al., 2012).

Benchikh and Louailèche (2014) showed that solvents (acetone, ethanol, methanol and water) at concentration $(40-100 \%)$ with solid-to-solvent ratio $(15 / 10$ to $75 / 10 \mathrm{mg} / \mathrm{ml})$ at extraction time $(60$ $120 \mathrm{~min})$ and extraction temperature $\left(25-90^{\circ} \mathrm{C}\right) \mathrm{had}$ statistically significant effects on phenolic compounds extracted from carob pulp and antioxidant activities. The best extraction conditions were $70 \%$ acetone, $25 \mathrm{mg} / 10 \mathrm{ml}, 90 \mathrm{~min}$ and $90^{\circ} \mathrm{C}$. Also, phenolic compounds content was positively correlated with antioxidant activities. Sebai et al (2013) showed that the carob polar extracts were richer in total polyphenols, total flavonoids and condensed tannins than the nonpolar extracts with quantitative variation of phenolic compounds between seeds and pulp.

Hussein et al. (2011) reported that the water extract of carob had antioxidative, antibacterial and antifungal activities against some pathogenic bacteria (Escherichia coli Staphylococcus auraus, Enterococcus feacalis, Bacillus subtilis and $B$. megaterium) and some yeast (Debaryomyces hansenii, Zygosaccharo mycesrouxii, Rhodotorula rubra, Candida shehatae and Candida tropicalis). Aissani et al. (2012) found that methanolic extract of carob leaves inhibited the growth of Listeria monocytogenes at $28.12 \mu \mathrm{g} / \mathrm{mL}$. The effect of this bacteriostatic concentration on the growth of this bacterium revealed a pattern of inhibition characterized by (a) resumed growth phase, which showed a lower rate of growth if compared with controls; and (b) first a lag and then a stationary phase at a lower bacterium concentration. Abd Razik et al. (2012) reported that the methanol extract of Ceratonia Siliqua had antibacterial activity on Gram positive bacteria (Lactobacillus sp. and Staphylococcus aureus) and Gram negative bacteria 
(Proteus sp., Pseudomonas aeruginosa, Escherichia coli and Enterococcus sp.). The extract produced inhibition zones against Gram positive bacteria sensitive to concentration ranged from 500 - 1000 $(\mathrm{mg} / \mathrm{ml})$ and Gram negative bacteria sensitive to concentration ranged from 125-1000 (mg/ml), Escherichia coli and Enterococcus sp. sensitive to concentration ranged from 1000-500 (mg/ml). Solvent (negative control) used for preparation different concentrations showed no activity against any tested bacteria.

Zunft et al. (2003) reported that carob fiber consumption lowered triglycerides in females by $11.3 \%$. Lipid lowering effects were more pronounced in females than in males. The consumption of carob fiber reduced LDL cholesterol by $10.5 \%$. The LDL: HDL cholesterol ratio was marginally decreased by $7.9 \%$ in the carob fiber group compared to the placebo group. Roso et al. (2010) found that the carob fiber rich in polyphenols reduced the total cholesterol by $17.8 \%$, LDL cholesterol by $22.5 \%$, LDL: HDL cholesterol ratio by $26.2 \%$ and triglycerides by $16.3 \%$ at the end of the study. No significant differences were found in the glucose, creatinine, uric acid, bilirubin, alkaline phosphatase, AST, and ALT) between the beginning and end of treatment. Mokhtari et al.(2011) stated that in diabetic adult male wistar rats received 150 , 300 and $600 \mathrm{mg} / \mathrm{kg}$ hydro-alcoholic seed extract of Ceratonia siliqua, the concentration of glucose, total cholesterol and LDL-C decreased significantly in respect to diabetic control group while, triglyceride level was only declined in group received $200 \mathrm{mg} / \mathrm{kg}$ extract. In addition, the serum level of HDL-C showed a considerable elevation. Ali et al. (2012) reported that rats feeding on high fructose diet led to significant increment in cholesterol, triglycerides, LDL-c, vLDL-c, uric acid, urea nitrogen and creatinine and decrement in HDL-c. Feeding rats on high fructose diet with the different levels of carob $(2.5 \%, 5 \%$ and $7.5 \%)$ improved all parameters and kidney weight, especially when used the high level from carob.

The aim of this investigation is to study the best method for extracting the antioxidants of carob, evaluate carob extracts as antimicrobial and evaluate carob water extract and fiber biological effects on diabetic and hypercholesterolemia rats.

\section{Materials and methods:}

\section{Plant Material}

Carob (Ceratonia siliqua L) was obtained from Horticulture Research Institute, Agriculture Research Center, Giza, Egypt (August 2013).

\section{Preparation of the samples:}

Carob pods were cleaned, dried and ground to fine powder.

\section{Preparation of carob fiber:}

Sugars and soluble tannins were removed from carob powder by two sequentially water extractions $\left(15 \mathrm{~min}\right.$ at $60^{\circ} \mathrm{C}$ followed by $30 \mathrm{~min}$ at $\left.110^{\circ} \mathrm{C}\right)$. The residue was washed and dried at $45^{\circ} \mathrm{C}$ under vacuum and reground (Wursch, 1979).

\section{Proximate analyses}

Moisture, ash, crude protein, crude fiber and total lipids contents were determined in carob according to A.O.A.C. (2005).Total hydrolysable carbohydrate was determined according to Dubois $\boldsymbol{e t}$ al. (1956).

\section{Extraction methods}

Water, methanol and ethanol were used at $25 \mathrm{C}$ for $24 \mathrm{~h}$, at $50^{\circ} \mathrm{C}$ for $20 \mathrm{~min}$ and by using Soxhelt apparatus also ,boiling water for 5, 10, 20 min were used to identify the most suitable solvent for the extraction of polyphenol, tannins, flavonoids and antioxidant. All extracts were then passed through filter paper and dried in oven at $50^{\circ} \mathrm{C}$.

\section{Chemical composition}

Total polyphenols content was estimated by the Folin-Ciocalteu method reported by Elfalleh $\boldsymbol{e t}$ al. (2009). Hydrolysable tannins content was determined by the method of Çam and Hişil (2010). The amount of total flavonoids in the extracts was measured spectrophotometrically by the method of Djeridane et al. (2006). The scavenging activity on DPPH radical of different extracts was determined according to the method reported by Okonogi $\boldsymbol{e t}$ al. (2007).

\section{Bacterial and fungal isolates}

Clinical isolates of $E$.coli NRRL B/210, Staph. aeureus NRRL B/3 B , Bacillus cereus NRRL B / G 43 , Bacillus megtarin NRRL B/1366, Listeria monocytogenase serotype NRRL Y/477, Klabsila pneumonia ATTCC700603 , Candida albicans NRRL Y/477, and Aspergillus niger NRRL/3 and Salmonella typhi ATTC5647006 were obtained from chemical department of Natural and Microbial product, National Research Center, and were kept in the laboratory in the frozen state until used. The antimicrobial effect of the carob extracts was evaluated using disk inhibition zone by the method described by Orak et al. (2011).

\section{Biological evaluation of carob water extracts and fiber.}

a. Experimental animals.

A total of 45 of adult's male albino rats (Wister Strain) weighed $200 \mathrm{~g}$ were obtained from Organization of Biological Products and Vaccines from Helwan breeding farm, Cairo, Egypt. The rats were housed in stain lasted cages with wire mesh bottoms in a room temperature maintained at $25{ }^{\circ} \mathrm{C} \pm$ $2^{\circ} \mathrm{C}$. Rats were kept under normal healthy conditions 
for one week and fed on basal diet. The diet contained Casein 10\%, Corn oil 10\%, Salt mixture $4 \%$, Vitamin mixture $1 \%$, Cellulose $5 \%$ and starch 70\% (Reeves et al. 1993).

Dosage and administration of decoction: The decoction was administered at a dosage of carob fiber $15 \%(15 \%$ from the starch of basil diet was substituted by $15 \%$ carob fiber) (Forestieri et al. 2006) and carob water extract $600 \mathrm{mg} / \mathrm{kg} /$ day (Mokhtari et al. 2011), using a Sondi needle by gastric gavage method ( Iddamaldeniya et al. 2006).

After that the rats were divided into three groups, 15 rats each. The first main group (control) was divided into three subgroups (5rats each) the first (control) was fed with basil diet for another 6 weeks. The second was fed on basil diet and administered of carob fiber The third was fed on basil diet and administered of carob water extract.

The second main group was the diabetic group. The rats were injected with a single dose of alloxan solution $150 \mathrm{mg} \backslash \mathrm{kg}$ body weight (Buko et al 1996). After 24hours of alloxan injection, the presence of diabetes was confirmed (glucose blood was higher than $180 \mathrm{mg} / \mathrm{dl}$ ). Rats were left for one week without any treatment to stabilize diabetes, and then were divided into three subgroups. The first (control diabetic) fed with basil diet for another 6 weeks. The second was fed on basil diet and administered of carob fiber. The third was fed on basil diet and administered of carob water extract.

The third main group was hypercholesterolemia group. The rats were fed on high fat diet similar to the control diet but differed in more fat content which was $10 \%$ sheep fat, $2 \%$ cholesterol and $0.25 \%$ bile salts and starch $57.75 \%$. for 2 weeks (Abdel-Rahim et al 2013), and then were divided into three subgroups. The first (control hypercholesterolemia) fed with basil diet for another 6 weeks. The second was fed on basil diet and administered of carob fiber. The third was fed on basil diet and administered of carob water extract.

\section{Blood sample}

At the end of experiment blood was collected in tubes from retro-orbital vein in two separated tubes, one tube with EDTA ( ethylene diamin tetra acetic acid) for determination of haematological parameters , and the other tube was centrifuged at $3000 \mathrm{rpm}$ for $20 \mathrm{~min}$, for serum preparation.

\section{Serum analysis}

Serum parameters were determined by enzymatic colorimeteric methods, glucose was determined according to the procedure of Trinder (1969). Serum triglyceride and total cholesterol were determined according to the methods of Fossati and Prencipe (1982) and Allain et al. (1974). Low density lipoprotein (LDL-cholesterol) and high density lipoprotein (HDL - cholesterol) were determined according to the method of Tietz (1976 a). Total bilirubin, total protein and albumin in serum were determined according to the methods of Walters and Gerarde (1970); Vassault et al. (1986) and Young et al. (1975). Alkaline phosphatases (ALP) was determined according to the method of Young et al. (1972). Serum aspartate transferease (AST) and alanine transferease (ALT) activities were measured colorimetrically according to the method of Tietz (1976 b). Serum urea, Uric acid and Creatinine were determined according to Tietz (1990), Vassault et al. (1986) and Tietz (1986).

\section{Haematology}

The red blood cells (RBC), white blood cells (WBC), and the hemoglobin $(\mathrm{Hb})$, were determined in Mindray 2800 hematology analyzer.

\section{Statistical analysis.}

Statistical analysis was done by Duncan's Methods (SAS, 1996).

\section{Results and discussion}

Data reported in Table (1) show the chemical composition of carob.

Table 1. Chemical composition of carob.

\begin{tabular}{ll}
\hline Constituents & $\begin{array}{l}\text { carob } \\
\text { based on dried weight }(\%)\end{array}$ \\
\hline Moisture & 11.8 \\
Crude fiber & 10.03 \\
Ash & 3.42 \\
Crude protein & 3.80 \\
Total lipid & 2.80 \\
Total carbohydrates & 70.97 \\
\hline
\end{tabular}

Data in Table (1) show that carob contained $11.8 \%$ moisture, $10.03 \%$ crude fiber, $3.42 \%$ ash, $3.80 \%$ crude protein, $2.80 \%$ total lipid and 70.97 total carbohydrates.

Khlifa et al. (2013) reported that the protein content in carob was $2.74 \%$ while, Avallon et al. (1997) reported that carob contained was $3 \%$ protein. Hussein et al. (2011) stated that carob contained $10.1 \%$ moisture, $5.48 \%$ total lipid, $73.14 \%$ total carbohydrate.

\section{Extraction.}

Table (2) presents the effect of extraction methods on the total phenols, total tannins, total flavonoids and antioxidants activity of carob extract. The results indicate that water extract at $50^{\circ} \mathrm{C}$ for 20 min recorded the highest value in total polyphenols, followed by methanol extract at $50^{\circ} \mathrm{C}$ for $20 \mathrm{~min}$. Total tannins recorded the highest value in water extract at $50^{\circ} \mathrm{C}$ for $20 \mathrm{~min}$, followed by methanol extract at $50^{\circ} \mathrm{C}$ for $20 \mathrm{~min}$, water and methanol extracts at $25^{\circ} \mathrm{C}$ for $24 \mathrm{~h}$. Water extract at $50^{\circ} \mathrm{C}$ gave the highest yield of total flavonoids, followed by 
water extract at $25{ }^{\circ} \mathrm{C}$ for $24 \mathrm{~h}$. Antioxidant activity was significantly highest in methanol extract at $50^{\circ} \mathrm{C}$ for $20 \mathrm{~min}$ followed by methanol extract at $25^{\circ} \mathrm{C}$ for $24 \mathrm{~h}$. Increasing water boiling time cause significant decrement in total polyphenols, total tannins, total flavonoids and antioxidants. Extraction by Soxhlet showed the lowest efficiency.
Al-Rawahi et al. (2013) indicated that water (as the highest polar solvent), extracted highest phenolic compounds followed by methanol then ethanol (due to decreasing polarity).

The obtained results are in agreement with those reported by Yim et al. (2009), Sebai et al. (2013), Zam et al. (2013), Abugri and McElhenney (2013) and Yaser et al. (2014).

Table 2. Effect of different methods of extraction of carob on total phenolic, total tannins, total flavonoids and antioxidantes activity.

\begin{tabular}{lllll}
\hline Extraction methods & $\begin{array}{l}\text { Total phenols } \\
(\mathbf{g} / \mathbf{1 0 0 g})\end{array}$ & $\begin{array}{l}\text { Total tannins } \\
(\mathbf{g} / \mathbf{1 0 0 g})\end{array}$ & $\begin{array}{l}\text { Total } \\
\text { flavonoids } \\
(\mathbf{m g} / \mathbf{1 0 0 g})\end{array}$ & $\begin{array}{l}\text { Antioxidants } \\
\text { activity \% }\end{array}$ \\
\hline Water extract at $25^{\circ} \mathrm{C}$ for $24 \mathrm{~h}$ & $2.103 \mathrm{~d} \pm 0.08$ & $0.85 \mathrm{~b} \pm 0.25$ & $24.20 \mathrm{~b} \pm 1.26$ & $63.89 \mathrm{e} \pm 1.2$ \\
Methanol extract at $25^{\circ} \mathrm{C}$ for $24 \mathrm{~h}$ & $1.75 \mathrm{e} \pm 0.11$ & $0.80 \mathrm{~b} \pm 0.21$ & $19.01 \mathrm{~d} \pm 1.87$ & $82.99 \mathrm{~b} \pm 2.73$ \\
Ethanol extract at $25^{\circ} \mathrm{C}$ for $24 \mathrm{~h}$ & $1.437 \mathrm{f} \pm 0.07$ & $0.396 \mathrm{c} \pm 0.15$ & $16.73 \mathrm{e} \pm 0.66$ & $69.32 \mathrm{~d} \pm 1.79$ \\
Water extract at $50^{\circ} \mathrm{C}$ for $20 \mathrm{~min}$ & $3.65 \mathrm{a} \pm 0.22$ & $1.20 \mathrm{a} \pm 0.19$ & $27.05 \mathrm{a} \pm 1.73$ & $69.13 \mathrm{~d} \pm 1.16$ \\
Methanol extract at $50^{\circ} \mathrm{C}$ for $20 \mathrm{~min}$ & $3.297 \mathrm{~b} \pm 0.20$ & $0.91 \mathrm{~b} \pm 0.29$ & $21.57 \mathrm{c} \pm 0.64$ & $86.00 \mathrm{a} \pm 1.51$ \\
Ethanol extract at $50^{\circ} \mathrm{C}$ for $20 \mathrm{~min}$ & $2.670 \mathrm{c} \pm 0.09$ & $0.42 \mathrm{c} \pm 0.15$ & $14.05 \mathrm{f} \pm 1.45$ & $74.28 \mathrm{c} \pm 1.66$ \\
Boiling water for $5 \mathrm{~min}$ & $1.12 \mathrm{~g} \pm 0.12$ & $0.37 \mathrm{dg} \pm 0.02$ & $18.84 \mathrm{~d} \pm 0.94$ & $64.16 \mathrm{e} \pm 3.56$ \\
Boiling water for $10 \mathrm{~min}$ & $0.87 \mathrm{~h} \pm 0.11$ & $0.32 \mathrm{~cd} \pm 0.02$ & $15.83 \mathrm{ef} \pm 0.87$ & $59.46 \mathrm{f} \pm 1.26$ \\
Boiling water for $20 \mathrm{~min}$ & $0.63 \mathrm{i} \pm 0.10$ & $0.253 \mathrm{de} \pm 0.02$ & $12.03 \mathrm{~g} \pm 1.52$ & $51.13 \mathrm{~g} \pm 0.27$ \\
water extract in Soxhlet & $0.11 \mathrm{j} \pm 0.01$ & $0.056 \mathrm{f} \pm 0.01$ & $7.10 \mathrm{i} \pm 0.42$ & $39.86 \mathrm{i} \pm 1.88$ \\
Methanol Soxhlet extract & $0.22 \mathrm{j} \pm 0.01$ & $0.46 \mathrm{ef} \pm 0.03$ & $10.15 \mathrm{gh} \pm 1.32$ & $44.01 \mathrm{~h} \pm 3.96$ \\
Ethanol Soxhlet extract & $0.12 \mathrm{j} \pm 0.01$ & $0.25 \mathrm{de} \pm 0.02$ & $8.31 \mathrm{hi} \pm 1.22$ & $43.41 \mathrm{~h} \pm 2.80$ \\
\hline
\end{tabular}

$a, b, c, \ldots . .$. means with column with differ letters different significantly $\quad(p \leq 0.05)$ from each other means followed by the same letter don't differ at 0.05 probability level.

\section{Antimicrobial effects:-}

Antimicrobial effects of carob extracts on some Gram positive bacteria.

Data in Table(3) show the effect of carob extracts against Gram positive bacteria; Staph.aeureus, Listeria monocytogenase, Bacillus cereus and Bacillus megaterium.

\section{Staph. aeureus}

Ethanol extract at $50^{\circ} \mathrm{C}$ for $20 \mathrm{~min}$, boiling water for $5 \mathrm{~min}$ and methanol extract at $50^{\circ} \mathrm{C}$ for 20 min had the highest antimicrobial effect. Boiling water for $20 \mathrm{~min}$ and water, methanol and ethanol shoxhlet extracts showed no antimicrobial effects.

\section{Listeria monocytogenase}

Ethanol extract at $50^{\circ} \mathrm{C}$ for $20 \mathrm{~min}$ had the highest antimicrobial effect followed by methanol extracts at $50{ }^{\circ} \mathrm{C}$ for $20 \mathrm{~min}$ and at $25{ }^{\circ} \mathrm{C}$ for $24 \mathrm{~h}$. Other extracts had no effect.

\section{Bacillus cereus.}

Water extract at $50^{\circ} \mathrm{C}$ for $20 \mathrm{~min}$, boiling water for $5 \mathrm{~min}$ and methanol extract at $50^{\circ} \mathrm{C}$ for 20 min had the highest inhibition zone $(13.5,13.33$ and $12.33 \mathrm{~mm})$.

\section{Bacillus megaterium.}

Methanol extract at $50{ }^{\circ} \mathrm{C}$ for $20 \mathrm{~min}$ and methanol soxhelt extract had the highest inhibition zones ( 11.17 and $10.17 \mathrm{~mm}$ ), followed by boiling water for 5 min and ethanol soxhelt extract.

The obtained results are in agreement with those reported Hussein et al. (2011), Abd Razik et al. (2012) and Hsouna et al .(2012).

E .coli.

Only water extract at $50^{\circ} \mathrm{C}$ for $20 \mathrm{nim}$ and 25 ${ }^{\circ} \mathrm{C}$ for $24 \mathrm{~h}$ showed antimicrobial effect against E.coli (10.17 and $8.33 \mathrm{~mm})$.

\section{Klebsila pneumonia}

Boiling water for $5 \mathrm{~min}$ and methanol Soxhlet extract had the highest antimicrobial effects. Boiling water for $20 \mathrm{~min}$ had no antimicrobial effect.

Salmonella typi.

All carob extracts had no antimicrobial effect against Salmonella typi.

The obtained results are in agreement with those reported by Kivcak et al (2002), Hussein et al (2011), Abd Razik et al (2012), Aissani et al (2012) and Hsouna et al (2012). 
Table 3. Effect of carob extracts on some Gram positive bacteria.

\begin{tabular}{|c|c|c|c|c|c|c|c|c|c|c|c|c|}
\hline \multirow[t]{3}{*}{ Treatments } & \multicolumn{3}{|c|}{ Staph. aeureus } & \multirow{2}{*}{\multicolumn{3}{|c|}{$\begin{array}{l}\text { Listeria monocytogenase } \\
\text { inhibition zone }(\mathrm{mm})\end{array}$}} & \multicolumn{3}{|c|}{ Bacillus cereus } & \multicolumn{3}{|c|}{ Bacillus megaterium } \\
\hline & & & & & & & & & & & & \\
\hline & $20 \mu$ & $40 \mu$ & mean & $20 \mu$ & $40 \mu$ & Mean & $20 \mu$ & $40 \mu$ & mean & $20 \mu$ & $40 \mu$ & mean \\
\hline Water extract at $25^{\circ} \mathrm{C}$ for $24 \mathrm{~h}$ & $\begin{array}{l}7.33 \mathrm{~g} \\
\pm 1.25\end{array}$ & $\begin{array}{l}9.66 \mathrm{cf} \\
\pm 0.8\end{array}$ & $8.5 \mathrm{~B}$ & $0 \mathrm{~d}$ & $0 \mathrm{~d}$ & $0 \mathrm{C}$ & Of & Of & 0D & $0 \mathrm{~g}$ & $0 \mathrm{~g}$ & $0 \mathrm{G}$ \\
\hline ethanol extract at $25^{\circ} \mathrm{C}$ for $24 \mathrm{~h}$ & $0 \mathrm{~h}$ & $\begin{array}{l}10.33 \mathrm{ce} \\
\pm 2.3\end{array}$ & $5.17 \mathrm{C}$ & od & $0 \mathrm{~d}$ & $0 \mathrm{C}$ & of & of & 0D & $0 \mathrm{~g}$ & $\begin{array}{c}8.33 \mathrm{ce} \\
\pm 2.11\end{array}$ & $4.17 \mathrm{EF}$ \\
\hline Methanol extract at $25^{\circ} \mathrm{C}$ for $24 \mathrm{~h}$ & $0 \mathrm{~h}$ & $\begin{array}{l}8.67 \mathrm{eg} \\
\pm 1.5\end{array}$ & $4.33 \mathrm{C}$ & $\begin{array}{l}7.0 \mathrm{bc} \\
\pm 1.10\end{array}$ & $\begin{array}{l}8.33 \mathrm{~b} \\
\pm 0.54\end{array}$ & $7.67 \mathrm{~B}$ & of & of & OD & $0 \mathrm{~g}$ & $\begin{array}{c}11.67 \mathrm{ab} \\
\pm 1.40\end{array}$ & 5.83DE \\
\hline Water extract at $50^{\circ} \mathrm{C}$ for $20 \mathrm{~min}$ & $\begin{array}{l}8.33 \mathrm{fg} \\
\pm 0.90\end{array}$ & $\begin{array}{l}8.67 \mathrm{eg} \\
\pm 0.7\end{array}$ & $8.5 \mathrm{~B}$ & $0 \mathrm{~d}$ & $0 \mathrm{~d}$ & $0 \mathrm{C}$ & $\begin{array}{l}11.33 \mathrm{de} \\
\pm 1.76\end{array}$ & $\begin{array}{l}15.67 \mathrm{a} \\
\pm 2.70\end{array}$ & $13.5 \mathrm{~A}$ & $0 \mathrm{~g}$ & $0 \mathrm{~g}$ & $0 \mathrm{G}$ \\
\hline Ethanol extract at $50^{\circ} \mathrm{C}$ for $20 \mathrm{~min}$ & $\begin{array}{l}11.0 \mathrm{~cd} \\
\pm 1.30\end{array}$ & $\begin{array}{l}13.00 \mathrm{ab} \\
\pm 1.8\end{array}$ & $12.0 \mathrm{~A}$ & $\begin{array}{l}8.33 \mathrm{~b} \\
\pm 1.00\end{array}$ & $\begin{array}{l}10.33 \mathrm{a} \\
\pm 1.20\end{array}$ & $9.33 \mathrm{~A}$ & $\begin{array}{l}9.0 \mathrm{de} \\
\pm 1.8\end{array}$ & $\begin{array}{c}13.67 \mathrm{ac} \\
\pm 3.10\end{array}$ & $11.33 \mathrm{AB}$ & $0 \mathrm{~g}$ & $\begin{array}{l}12.67 \mathrm{ab} \\
\pm 1.15\end{array}$ & 6.33CD \\
\hline Methanol extract at $50^{\circ} \mathrm{C}$ for $20 \mathrm{~min}$ & $\begin{array}{l}10.67 \mathrm{~cd} \\
\pm 1.50\end{array}$ & $\begin{array}{l}11.33 \mathrm{bc} \\
\pm 2.1\end{array}$ & $11.0 \mathrm{~A}$ & $\begin{array}{l}6.0 \mathrm{c} \\
\pm 0.50\end{array}$ & $\begin{array}{l}10.33 \mathrm{a} \\
\pm 1.51\end{array}$ & $8.17 \mathrm{AB}$ & $\begin{array}{l}10.67 \mathrm{be} \\
\pm 1.60\end{array}$ & $\begin{array}{l}14.0 \mathrm{ab} \\
\pm 2.00\end{array}$ & $12.33 \mathrm{~A}$ & $\begin{array}{l}8.67 \mathrm{ce} \\
\pm 0.65\end{array}$ & $\begin{array}{l}13.67 \mathrm{a} \\
\pm 0.86\end{array}$ & $11.17 \mathrm{~A}$ \\
\hline Boiling water for $5 \mathrm{~min}$ & $\begin{array}{l}10.0 \mathrm{cf} \\
\pm 1.20\end{array}$ & $\begin{array}{l}13.67 \mathrm{a} \\
\pm 0.76\end{array}$ & $11.83 \mathrm{~A}$ & $0 \mathrm{~d}$ & od & $0 \mathrm{C}$ & $\begin{array}{l}10.33 \mathrm{ce} \\
\pm 0.87\end{array}$ & $\begin{array}{r}16.33 \mathrm{a} \\
\pm 3.00\end{array}$ & $13.33 \mathrm{~A}$ & $\begin{array}{l}7.67 \mathrm{df} \\
\pm 1.15\end{array}$ & $\begin{array}{l}11.00 \mathrm{ac} \\
\pm 1.73\end{array}$ & $9.33 \mathrm{AB}$ \\
\hline Boiling water for10 min & $\begin{array}{l}7.33 \mathrm{~g} \\
\pm 1.15 \\
\end{array}$ & $\begin{array}{l}9.33 \mathrm{df} \\
\pm 0.54 \\
\end{array}$ & 9.33DF & od & $0 \mathrm{~d}$ & $0 \mathrm{C}$ & Of & $\begin{array}{r}12.0 \mathrm{bd} \\
\pm 1.20 \\
\end{array}$ & $6.00 \mathrm{C}$ & $0 \mathrm{~g}$ & $\begin{array}{l}6.33 \mathrm{ef} \\
\pm 0.63 \\
\end{array}$ & $3.17 \mathrm{~F}$ \\
\hline Boiling water for $20 \mathrm{~min}$ & $0 \mathrm{~h}$ & $0 \mathrm{~h}$ & $0 \mathrm{D}$ & $0 \mathrm{~d}$ & Od & $0 \mathrm{C}$ & of & Of & 0D & $0 \mathrm{~g}$ & $0 \mathrm{~g}$ & $0 \mathrm{~g}$ \\
\hline Water Soxhlet extract & $\mathrm{Oh}$ & $\mathrm{Oh}$ & 0D & $0 \mathrm{~d}$ & $0 \mathrm{~d}$ & $0 \mathrm{C}$ & $\begin{array}{l}9.0 \mathrm{de} \\
\pm 0.54 \\
\end{array}$ & $\begin{array}{l}10.33 \mathrm{ce} \\
\pm 1.4 \\
\end{array}$ & $9.67 \mathrm{~B}$ & $\begin{array}{l}5.33 \mathrm{f} \\
\pm 1.12 \\
\end{array}$ & $\begin{array}{c}11.00 \mathrm{ac} \\
\pm 1.76 \\
\end{array}$ & $8.17 \mathrm{BC}$ \\
\hline Ethanol Soxhlet extract & $0 \mathrm{~h}$ & $0 \mathrm{~h}$ & 0D & od & $0 \mathrm{~d}$ & $\overline{0 \mathrm{C}}$ & $\begin{array}{l}8.33 \mathrm{e} \\
\pm 1.3 \\
\end{array}$ & $\begin{array}{l}10.67 \mathrm{be} \\
\pm 2.00\end{array}$ & $9.50 \mathrm{~B}$ & $\begin{array}{l}8.33 \mathrm{ce} \\
\pm 0.54\end{array}$ & $\begin{array}{l}10.33 \mathrm{bd} \\
\pm 0.67\end{array}$ & $9.33 \mathrm{AB}$ \\
\hline Methanol Soxhlet extract & $0 \mathrm{~h}$ & $0 \mathrm{~h}$ & $0 \mathrm{D}$ & $0 \mathrm{~d}$ & $0 \mathrm{~d}$ & $0 \mathrm{C}$ & $\begin{array}{l}11.33 \mathrm{be} \\
\pm 1.8 \\
\end{array}$ & $\begin{array}{l}11.67 \mathrm{be} \\
\pm 0.9\end{array}$ & $11.5 \mathrm{AB}$ & $\begin{array}{l}8.67 \mathrm{ce} \\
\pm 0.86 \\
\end{array}$ & $\begin{array}{l}11.67 \mathrm{ab} \\
\pm 1.54 \\
\end{array}$ & $10.17 \mathrm{~A}$ \\
\hline Mean conc & $4.56 \mathrm{~B}$ & $7.05 \mathrm{~A}$ & & $1.68 \mathrm{~B}$ & $2.42 \mathrm{~A}$ & & $6.02 \mathrm{~B}$ & $8.5 \mathrm{~A}$ & & $3.22 \mathrm{~B}$ & $8.05 \mathrm{~A}$ & \\
\hline
\end{tabular}


Table 4. Effect of carob extracts on some Gram negative bacteria.

\begin{tabular}{|c|c|c|c|c|c|c|}
\hline \multirow[t]{3}{*}{ Treatments } & \multicolumn{3}{|l|}{ E.coli } & \multicolumn{3}{|c|}{ Klebsila pneumonia. } \\
\hline & \multicolumn{6}{|c|}{ inhibition zone $(\mathrm{mm})$} \\
\hline & $20 \mu$ & $40 \mu$ & mean & $20 \mu$ & $40 \mu$ & mean \\
\hline Water extract at $25^{\circ} \mathrm{C}$ for $24 \mathrm{~h}$ & $\begin{array}{l}8.00 \mathrm{~b} \\
\pm 1.00 \\
\end{array}$ & $\begin{array}{r}8.667 \mathrm{~b} \\
\pm 1.50 \\
\end{array}$ & $8.33 \mathrm{~B}$ & $\begin{array}{l}8.00 \mathrm{df} \\
\pm 1.9 \\
\end{array}$ & $\begin{array}{l}8.66 \mathrm{cf} \\
\pm 1.50 \\
\end{array}$ & $8.83 \mathrm{CE}$ \\
\hline Ethanol extract at $25^{\circ} \mathrm{C}$ for $24 \mathrm{~h}$ & $0 \mathrm{c}$ & $0 \mathrm{c}$ & OC & $\begin{array}{l}6.667 \mathrm{ef} \\
\pm 1.33 \\
\end{array}$ & $\begin{array}{l}12.00 \mathrm{ac} \\
\pm 2.00\end{array}$ & $9.33 \mathrm{CE}$ \\
\hline Methanol extract at $25^{\circ} \mathrm{C}$ for $24 \mathrm{~h}$ & $0 \mathrm{c}$ & $0 \mathrm{c}$ & $0 \mathrm{C}$ & $\begin{array}{l}6.33 \mathrm{f} \\
\pm 0.40 \\
\end{array}$ & $\begin{array}{l}9.667 \mathrm{bf} \\
\pm 1.00 \\
\end{array}$ & $8.00 \mathrm{E}$ \\
\hline Water extract at $50^{\circ} \mathrm{C}$ for $20 \mathrm{~min}$ & $\begin{array}{l}9.00 \mathrm{~b} \\
\pm 0.50\end{array}$ & $\begin{array}{r}11.33 \mathrm{a} \\
\pm 1.40 \\
\end{array}$ & $10.17 \mathrm{~A}$ & $\begin{array}{l}6.33 \mathrm{ef} \\
\pm 1.00 \\
\end{array}$ & $\begin{array}{l}11.33 \mathrm{bd} \\
\pm 1.73 \\
\end{array}$ & $8.83 \mathrm{CE}$ \\
\hline Ethanol extract at $50^{\circ} \mathrm{C}$ for $20 \mathrm{~min}$ & $0 \mathrm{c}$ & $0 \mathrm{c}$ & $0 \mathrm{c}$ & $\begin{array}{l}\text { 10.33be } \\
\pm 1.1 \\
\end{array}$ & $\begin{array}{l}20.33 \mathrm{ac} \\
\pm 3.7 \\
\end{array}$ & $11.33 \mathrm{BC}$ \\
\hline Methanol extract at $50^{\circ} \mathrm{C}$ for $20 \mathrm{~min}$ & $0 \mathrm{c}$ & $0 \mathrm{c}$ & $0 \mathrm{C}$ & $\begin{array}{l}10.33 \mathrm{bc} \\
\pm 1.00\end{array}$ & $\begin{array}{l}12.33 \mathrm{ac} \\
\pm 1.51 \\
\end{array}$ & $11.33 \mathrm{BC}$ \\
\hline Boiling water for $5 \mathrm{~min}$ & $0 \mathrm{c}$ & $0 \mathrm{c}$ & OC & $\begin{array}{l}12.67 \mathrm{a} \mathrm{b} \\
\pm 1.15\end{array}$ & $\begin{array}{l}15.67 \mathrm{a} \\
\pm 2.08\end{array}$ & $14.17 \mathrm{~A}$ \\
\hline Boiling water for $10 \mathrm{~min}$ & $0 \mathrm{c}$ & $0 \mathrm{c}$ & $0 \mathrm{C}$ & $\begin{array}{l}10.00 \mathrm{bf} \\
\pm 1.25\end{array}$ & $\begin{array}{l}11.33 \mathrm{bd} \\
\pm 0.86 \\
\end{array}$ & $10.67 \mathrm{BD}$ \\
\hline Boiling water for $20 \mathrm{~min}$ & $0 \mathrm{c}$ & $0 \mathrm{c}$ & $0 \mathrm{C}$ & $0 \mathrm{~g}$ & $0 \mathrm{~g}$ & $\mathrm{OF}$ \\
\hline Water Soxhlet extract & $0 \mathrm{c}$ & $0 \mathrm{c}$ & $0 \mathrm{C}$ & $\begin{array}{l}8.66 \mathrm{cf} \\
\pm 0.62\end{array}$ & $\begin{array}{l}10.67 \mathrm{bd} \\
\pm 1.1\end{array}$ & $9.66 \mathrm{CE}$ \\
\hline Ethanol Soxhlet extract & $0 \mathrm{c}$ & $0 \mathrm{c}$ & $0 \mathrm{C}$ & $\begin{array}{l}12.67 \mathrm{ab} \\
\pm 1.06 \\
\end{array}$ & $\begin{array}{l}13.00 \mathrm{ab} \\
\pm 1.41\end{array}$ & $12.83 \mathrm{AB}$ \\
\hline Methanol Soxhlet extract & $0 \mathrm{c}$ & $0 \mathrm{c}$ & $0 \mathrm{C}$ & $\begin{array}{l}12.67 \mathrm{ab} \\
\pm 2.5\end{array}$ & $\begin{array}{l}15.67 \mathrm{a} \\
\pm 3.46 \\
\end{array}$ & $14.17 \mathrm{~A}$ \\
\hline Mean conc & $8.72 \mathrm{~B}$ & $11.06 \mathrm{~A}$ & & $1.41 \mathrm{~A}$ & $1.66 \mathrm{~A}$ & \\
\hline
\end{tabular}

a,b,c,..f means with column with differ letters differ significantly $\quad(\mathrm{p} \leq 0.05)$ from each other means followed by the same letter don't differ at 0.05 probability level. 
Data in Table (5) presents the effect of various carob extracts against Candida albicans and Aspergillus niger.

\section{Candida albicans}

Methanol Soxhelt extract and at $50{ }^{\circ} \mathrm{C}$ for $20 \mathrm{~min}$ had the highest antimicrobial effects. Water, ethanol and methanol extracts at $25{ }^{\circ} \mathrm{C}$ for $24 \mathrm{hr}$ and boiling water for $20 \mathrm{~min}$ had no antimicrobial effect.

\section{Aspergillus niger.}

Water, ethanol and methanol extracts at $50{ }^{\circ} \mathrm{C}$ for $20 \mathrm{~min}$ had the highest inhibition activity. Methanol extract at $25{ }^{\circ} \mathrm{C}$ for $24 \mathrm{~h}$ gave the lowest antimicrobial effect. The other extracts had no effect. The obtained results are in agreement with those of Kicvak et al (2002) and Hsouna et al (2012).

Table 5. Effect of carob extracts on some fungi

\begin{tabular}{|c|c|c|c|c|c|c|}
\hline \multirow[t]{2}{*}{ Treatments } & \multicolumn{3}{|c|}{ Candida albicans } & \multicolumn{3}{|c|}{ Aspergillus niger } \\
\hline & \multicolumn{3}{|c|}{ inhibition zone (mm) } & & & \\
\hline & $20 \mu$ & $40 \mu$ & mean & $20 \mu$ & $40 \mu$ & mean \\
\hline Water extract at $25^{\circ} \mathrm{C}$ for $24 \mathrm{~h}$ & $0 \mathrm{i}$ & $0 \mathrm{i}$ & $\mathrm{OF}$ & $0 \mathrm{e}$ & $0 \mathrm{e}$ & OD \\
\hline Ethanol extract at $25^{\circ} \mathrm{C}$ for $24 \mathrm{~h}$ & $0 \mathrm{i}$ & $0 \mathrm{i}$ & $\mathrm{OF}$ & $0 \mathrm{e}$ & $0 \mathrm{e}$ & OD \\
\hline Methanol extract at $25^{\circ} \mathrm{C}$ for $24 \mathrm{~h}$ & $0 \mathrm{i}$ & $0 \mathrm{i}$ & $0 \mathrm{~F}$ & $0 \mathrm{e}$ & $\begin{array}{l}3.66 \mathrm{~d} \\
\pm 1.00\end{array}$ & $1.83 \mathrm{C}$ \\
\hline Water extract at $50^{\circ} \mathrm{C}$ for $20 \mathrm{~min}$ & $\begin{array}{l}11.0 \mathrm{fg} \pm \\
1.52\end{array}$ & $\begin{array}{l}17.67 \mathrm{ac} \\
\pm 2.0\end{array}$ & 14.33B & $\begin{array}{l}11.00 \mathrm{~b} \\
\pm 2.5\end{array}$ & $\begin{array}{l}18.00 \mathrm{a} \\
\pm 1.51\end{array}$ & $14.50 \mathrm{~A}$ \\
\hline Ethanol extract at $50^{\circ} \mathrm{C}$ for $20 \mathrm{~min}$ & $\begin{array}{l}13.00 \mathrm{ef} \\
\pm 1.67\end{array}$ & $\begin{array}{r}15.33 \mathrm{~d} \\
\pm 3.10\end{array}$ & 14.17B & $\begin{array}{l}10.33 b c \\
\pm 1.40\end{array}$ & $\begin{array}{l}17.67 \mathrm{a} \\
\pm 0.89\end{array}$ & $14.00 \mathrm{~A}$ \\
\hline Methanol extract at $50^{\circ} \mathrm{C}$ for $20 \mathrm{~min}$ & $\begin{array}{l}14.33 \mathrm{de} \\
\pm 2.10\end{array}$ & $\begin{array}{r}19.33 \mathrm{a} \\
\pm 2.40\end{array}$ & $16.83 \mathrm{~A}$ & $\begin{array}{l}8.00 \mathrm{c} \\
\pm 1.15\end{array}$ & $\begin{array}{l}10.33 b c \\
\pm 2.08\end{array}$ & $9.16 \mathrm{~B}$ \\
\hline Boiling water for $5 \mathrm{~min}$ & $\begin{array}{l}9.0 \mathrm{gh} \quad \pm \\
1.00\end{array}$ & $\begin{array}{c}10.00 \mathrm{gh} \\
\pm 1.50\end{array}$ & $9.50 \mathrm{D}$ & $0 \mathrm{e}$ & $0 \mathrm{e}$ & $0 \mathrm{D}$ \\
\hline Boiling water for $10 \mathrm{~min}$ & Oi & $\begin{array}{l}8.67 \mathrm{~h} \\
\pm 0.54\end{array}$ & $4.33 \mathrm{E}$ & $0 \mathrm{e}$ & $0 \mathrm{e}$ & $0 \mathrm{D}$ \\
\hline Boiling water for $20 \mathrm{~min}$ & $0 \mathrm{i}$ & Oi & $\mathrm{OF}$ & $0 \mathrm{e}$ & $0 \mathrm{e}$ & OD \\
\hline Water Soxhlet extract & $\begin{array}{l}9.67 \mathrm{gh} \pm \\
1.52\end{array}$ & $\begin{array}{c}12.67 \mathrm{ef} \\
\pm 1.94\end{array}$ & 11.17C & $0 \mathrm{e}$ & $0 \mathrm{e}$ & $0 \mathrm{D}$ \\
\hline Ethanol Soxhlet extract & $\begin{array}{l}13.0 \text { ef } \pm \\
0.57\end{array}$ & $\begin{array}{l}16.00 \mathrm{~cd} \\
\pm 2.09\end{array}$ & 14.50B & $0 \mathrm{e}$ & $0 \mathrm{e}$ & OD \\
\hline Methanol Soxhlet extract & $\begin{array}{l}16.33 b d \\
\pm 1.52\end{array}$ & $\begin{array}{c}18.33 \mathrm{ab} \\
\pm 2.50\end{array}$ & $17.33 \mathrm{~A}$ & $0 \mathrm{e}$ & $0 \mathrm{e}$ & OD \\
\hline Mean & $7.19 \mathrm{~B}$ & $9.80 \mathrm{~A}$ & & $2.44 \mathrm{~B}$ & $4.13 \mathrm{~A}$ & \\
\hline
\end{tabular}

$\mathrm{a}, \mathrm{b}, \mathrm{c}, \ldots . . . \mathrm{i}$ means within column with different letters different significantly $(\mathrm{p} \leq 0.05)$ from each other means followed by the same letter don't differ at 0.05 probability level.

\section{Biological effects:-}

Data concerning the effect of carob fiber and water extract on blood serum glucose and lipid profile are shown in (Table, 6). Data reported in Table (6) indicate that glucose levels in diabetic and hypercholesterolemia groups had significant increment compared with control. Carob fiber and water extract administration caused significant decrement in glucose level comparing with diabetic and hypercholesterolemia control groups.

The obtained results are in agreement with those reported by Tabatabai and $\mathbf{L i}$ (2000), Forestieri et al. (2006), and Mokhtari et al. (2011).

Diabetic and hypercholesterolemia groups administrated with carob fiber and water extract showed significant decrement in triglyceride, total cholesterol and LDL levels and significant increment in HDL level in diabetic group comparing to diabetic and hypercholesterolemia control groups.
The obtained results are in agreement with those reported by Zunft et al. (2001), Zunft et al. (2003), Roso et al. (2010), Mokhtari et al. (2011) and Ali et al. (2012). Table (7) show the effect of orally intake carob fiber and water extract of carob on liver functions.

Diabetic group administrated with carob fiber and carob water extract showed non-significant difference in total bilirubin, total protein and albumin. Carob fiber administration caused significant decrement in ALT comparing to diabetic control group. Carob water extract showed significant decrement in AST, ALT and ALP comparing with diabetic control group. Hypercholesterolemia groups administrated with carob fiber and water extract showed no significant difference in total bilrubin and significant decrement in total protein, albumin, AST, ALT and ALP comparing with hypercholesterolemia control group. The obtained results are in a agreement with those 
reported by Roso et al (2010). Data in Table (8) extract on kidney functions. show the effect of orally intake carob fiber and water

Table 6. Effect of orally intake carob fiber and carob water extract on glucose and lipid profile.

\begin{tabular}{|c|c|c|c|c|c|c|c|c|}
\hline Groups & $\begin{array}{l}\text { Blood } \quad \text { serum } \\
\text { glucose } \mathrm{mg} / \mathrm{dl}\end{array}$ & $\begin{array}{l}\text { Triglyceride } \\
(\mathrm{mg} / \mathrm{dl})\end{array}$ & $\begin{array}{l}\text { Total } \\
\text { cholesterol } \\
(\mathrm{mg} / \mathrm{dl})\end{array}$ & & $\begin{array}{l}\mathrm{LDL} \\
(\mathrm{mg} / \mathrm{dl})\end{array}$ & & $\begin{array}{l}\text { HDL } \\
(\mathrm{mg} / \mathrm{dl})\end{array}$ & \\
\hline Control Basal diet & 81.09 ce \pm 11.00 & $61.46 \mathrm{df} \pm 4.0$ & $\begin{array}{l}100.30 \mathrm{e} \\
5.9\end{array}$ & \pm & $\begin{array}{l}39.25 f \\
1.60\end{array}$ & \pm & $\begin{array}{l}45.45 \mathrm{bd} \\
1.12\end{array}$ & \pm \\
\hline $\begin{array}{l}\text { Basal diet }+ \text { Carob } \\
\text { fiber }\end{array}$ & $61.84 \mathrm{e} \pm 12.76$ & $\begin{array}{ll}54.44 \mathrm{eg} & \pm \\
3.13 & \end{array}$ & $\begin{array}{l}78.27 \mathrm{~h} \\
3.66\end{array}$ & \pm & $\begin{array}{l}25.99 \mathrm{~g} \\
0.54\end{array}$ & \pm & $\begin{array}{l}46.94 \mathrm{bd} \\
\pm 1.84\end{array}$ & \\
\hline $\begin{array}{l}\text { Basal diet }+ \text { Carob } \\
\text { water extract }\end{array}$ & $63.85 \mathrm{e} \pm 10.38$ & $49.61 \mathrm{~g} \pm 2.14$ & $\begin{array}{l}79.98 \mathrm{gh} \\
3.41\end{array}$ & \pm & $\begin{array}{l}34.58 f g \\
2.15\end{array}$ & \pm & $\begin{array}{l}37.65 \mathrm{f} \\
3.30\end{array}$ & \pm \\
\hline Diabetic control group & $420.10 \mathrm{a} \pm 86.32$ & $91.87 \mathrm{~b} \pm 7.69$ & $\begin{array}{l}131.60 \mathrm{~b} \\
7.5\end{array}$ & \pm & $\begin{array}{l}68.24 \mathrm{c} \\
1.76\end{array}$ & \pm & $\begin{array}{l}29.03 \mathrm{~g} \\
0.97\end{array}$ & \pm \\
\hline $\begin{array}{l}\text { Basal diet }+ \\
\text { carob fiber }\end{array}$ & $116.30 b \pm 14.73$ & $\begin{array}{l}57.44 \mathrm{eg} \\
9.43\end{array}$ & $\begin{array}{l}120.00 \mathrm{c} \\
5.61\end{array}$ & \pm & $\begin{array}{l}55.3 \mathrm{de} \\
1.52\end{array}$ & \pm & $\begin{array}{l}48.94 \mathrm{bd} \\
3.33\end{array}$ & \pm \\
\hline $\begin{array}{l}\text { Basal diet }+ \\
\text { carob water extract }\end{array}$ & $123.8 b \pm 13.27$ & $\begin{array}{l}59.86 \mathrm{eg} \\
5.19\end{array}$ & $\begin{array}{l}116.40 \mathrm{~cd} \\
\pm 6.64\end{array}$ & & $\begin{array}{l}58.67 d \\
2.00\end{array}$ & \pm & $\begin{array}{l}43.43 \text { ce } \\
1.73\end{array}$ & \pm \\
\hline $\begin{array}{l}\text { Hypercholesterolemia } \\
\text { control group }\end{array}$ & $\begin{array}{l}102.00 \mathrm{bd} \\
16.40\end{array}$ & $\begin{array}{l}121.60 \mathrm{a} \\
16.67\end{array}$ & $\begin{array}{l}157.85 \mathrm{a} \\
4.78\end{array}$ & \pm & $\begin{array}{l}109.00 \mathrm{a} \\
3.40\end{array}$ & \pm & $\begin{array}{l}29.77 \mathrm{~g} \\
1.90\end{array}$ & \pm \\
\hline $\begin{array}{l}\text { Basal diet }+ \\
\text { carob fiber }\end{array}$ & $73.72 b e \pm 4.50$ & $\begin{array}{l}72.25 \mathrm{~cd} \\
6.36\end{array}$ & $\begin{array}{l}139.50 \mathrm{~b} \\
4.31\end{array}$ & \pm & $\begin{array}{l}85.62 b \\
0.76\end{array}$ & \pm & $\begin{array}{l}35.31 \mathrm{fg} \\
2.65\end{array}$ & \pm \\
\hline $\begin{array}{l}\text { Basal diet }+ \\
\text { carob water extract }\end{array}$ & $68.41 \mathrm{e} \pm 4.13$ & $\begin{array}{l}61.99 \mathrm{de} \\
5.01\end{array}$ & $\begin{array}{l}135.00 \mathrm{~b} \\
4.77\end{array}$ & \pm & $\begin{array}{l}89.45 b \\
0.58\end{array}$ & \pm & $\begin{array}{l}30.31 \mathrm{~g} \\
0.58\end{array}$ & \pm \\
\hline
\end{tabular}

a,b,c,....h means column with different letters differ significantly $(\mathrm{p} \leq 0.05)$ from each other means followed by the same letter don't differ the 0.05 probability level.

Each value presents the mean of 5 rats \pm S.E.

Table 7. Effect of orally intake carob fiber and carob water extract on liver functions.

\begin{tabular}{|c|c|c|c|c|c|c|}
\hline Groups & $\begin{array}{l}\text { Total } \\
\text { Bilirubin } \\
(\mathrm{mg} / \mathrm{dl})\end{array}$ & $\begin{array}{l}\text { Total } \\
\text { protein } \\
(\mathrm{g} \backslash \mathrm{l})\end{array}$ & $\begin{array}{l}\text { Albumin } \\
(\mathrm{g} / \mathrm{dl})\end{array}$ & $\begin{array}{l}\text { AST } \\
\text { U L-1 }\end{array}$ & $\begin{array}{l}\text { ALT } \\
\mathrm{UL}-1\end{array}$ & $\begin{array}{l}\text { ALP } \\
\text { U L-1 }\end{array}$ \\
\hline Control Basal diet & $\begin{array}{l}0.90 \mathrm{~b} \\
\pm 0.14\end{array}$ & $\begin{array}{l}6.95 \mathrm{~d} \pm \\
0.91\end{array}$ & $\begin{array}{l}2.78 \mathrm{e} \\
\pm 0.26\end{array}$ & $\begin{array}{l}24.85 f g \\
\pm 2.93\end{array}$ & $\begin{array}{l}34.09 \mathrm{gh} \\
\pm 1.19\end{array}$ & $\begin{array}{l}84.06 \mathrm{df} \pm \\
4.60\end{array}$ \\
\hline Basal diet + Carob fiber & $\begin{array}{l}1.76 \mathrm{ab} \\
0.09\end{array}$ & $\begin{array}{l}6.74 \mathrm{~d} \pm \\
0.32\end{array}$ & $\begin{array}{l}2.96 \mathrm{de} \\
0.23\end{array}$ & $\begin{array}{l}26.71 \mathrm{f} \\
\pm 2.87\end{array}$ & $\begin{array}{ll}36.24 \mathrm{~g} \quad \pm \\
1.76\end{array}$ & $\begin{array}{l}81.01 \mathrm{ef} \pm \\
4.95\end{array}$ \\
\hline Basal diet + Carob water extract & $\begin{array}{l}1.67 \mathrm{ab} \\
0.16\end{array}$ & $\begin{array}{l}6.62 \mathrm{~d} \pm \\
0.43\end{array}$ & $\begin{array}{l}2.97 \mathrm{de} \\
0.57\end{array}$ & $\begin{array}{l}26.98 \mathrm{f} \quad \pm \\
1.50\end{array}$ & $\begin{array}{l}32.91 \mathrm{gi} \pm \\
3.40\end{array}$ & $\begin{array}{l}75.59 \mathrm{f} \\
\pm 2.14\end{array}$ \\
\hline $\begin{array}{l}\text { Diabetic control group }+ \text { Basal } \\
\text { diet }\end{array}$ & $\begin{array}{l}2.10 \mathrm{a} \\
\pm 0.08\end{array}$ & $\begin{array}{l}6.93 \mathrm{~d} \pm \\
0.91\end{array}$ & $\begin{array}{l}3.51 \text { be } \\
0.41\end{array}$ & $\begin{array}{l}43.53 b \quad \pm \\
4.93\end{array}$ & $\begin{array}{ll}66.83 b & \pm \\
3.19\end{array}$ & $\begin{array}{l}98.69 \mathrm{c} \quad \pm \\
5.67\end{array}$ \\
\hline $\begin{array}{l}\text { Diabetic + Basal diet + Carob } \\
\text { fiber }\end{array}$ & $\begin{array}{l}1.62 \mathrm{ab} \\
0.11\end{array}$ & $\begin{array}{l}6.30 \mathrm{~d} \pm \\
0.20\end{array}$ & $\begin{array}{l}3.53 \mathrm{be} \\
0.77\end{array}$ & $\begin{array}{l}43.34 \mathrm{~b} \quad \pm \\
1.00\end{array}$ & $\begin{array}{l}58.29 \mathrm{~cd} \\
\pm 2.89\end{array}$ & $\begin{array}{l}90.54 \mathrm{~cd} \\
\pm 4.60\end{array}$ \\
\hline $\begin{array}{l}\text { Diabetic + Basal diet }+ \text { carob } \\
\text { water extract }\end{array}$ & $\begin{array}{l}1.57 \mathrm{ab} \\
0.04\end{array}$ & $\begin{array}{l}6.79 \mathrm{~d} \pm \\
0.63\end{array}$ & $\begin{array}{l}3.8 \mathrm{ab} \\
\pm 0.29\end{array}$ & $\begin{array}{l}38.38 \mathrm{~cd} \pm \\
2.99\end{array}$ & $\begin{array}{l}56.39 \mathrm{de} \\
\pm 2.00\end{array}$ & $\begin{array}{l}86.97 \mathrm{de} \\
\pm 5.30\end{array}$ \\
\hline $\begin{array}{ll}\text { Hypercholesterolemia } & \text { control } \\
\text { group }\end{array}$ & $\begin{array}{l}2.53 \mathrm{a} \\
\pm 0.23\end{array}$ & $\begin{array}{l}9.60 \mathrm{a} \pm \\
0.42\end{array}$ & $\begin{array}{l}4.53 \mathrm{a} \\
1.11 \\
\end{array}$ & $\begin{array}{l}56.26 \mathrm{a} \pm \\
1.40\end{array}$ & $\begin{array}{l}77.26 \mathrm{a} \\
\pm 4.90\end{array}$ & $\begin{array}{l}147.30 \mathrm{a} \\
\pm 8.40\end{array}$ \\
\hline $\begin{array}{l}\text { Hypercholesterolemia }+ \text { Basal } \\
\text { diet }+ \text { Carob fiber }\end{array}$ & $\begin{array}{l}1.82 \mathrm{ab} \\
0.15\end{array}$ & $\begin{array}{l}8.25 b \quad \pm \\
0.97\end{array}$ & $\begin{array}{l}3.78 b c \\
0.60\end{array}$ & $\begin{array}{l}44.60 \mathrm{~b} \quad \pm \\
1.75\end{array}$ & $\begin{array}{ll}68.83 b & \pm \\
3.01 & \end{array}$ & $\begin{array}{l}120.25 b \\
\pm 2.90\end{array}$ \\
\hline $\begin{array}{l}\text { Hypercholesterolemia }+ \text { Basal } \\
\text { diet }+ \text { carob water extract }\end{array}$ & $\begin{array}{l}1.77 \mathrm{ab} \\
0.08\end{array}$ & $\begin{array}{l}6.81 b \quad \pm \\
0.32\end{array}$ & $\begin{array}{l}3.75 b c \\
0.70\end{array}$ & $\begin{array}{l}41.65 b c \pm \\
4.50\end{array}$ & $\begin{array}{l}61.92 \mathrm{c} \quad \pm \\
2.00\end{array}$ & $\begin{array}{l}118.85 b \\
\pm 2.50\end{array}$ \\
\hline
\end{tabular}

$a, b, c, \ldots g$ means within column with different letters differ significantly $(p \leq 0.05)$ from each other means followed by the same letter don't differ at 0.05 probability level.

Each value represents the mean of 5 rats \pm S.E. 
Table 8. Effect of orally intake carob fiber and carob water extract on kidney functions.

\begin{tabular}{|c|c|c|c|}
\hline Groups & $\begin{array}{l}\text { Urea } \\
\mathrm{mg} / \mathrm{dl}\end{array}$ & $\begin{array}{l}\text { Uric acid } \\
\mathrm{mg} / \mathrm{dl}\end{array}$ & $\begin{array}{l}\text { Creatinine } \\
\mathrm{mg} / \mathrm{dl}\end{array}$ \\
\hline Control Basal diet & $50.97 \mathrm{fg} \pm 3.75$ & $2.81 \mathrm{de} \pm 0.15$ & $0.28 \mathrm{~cd} \pm 0.10$ \\
\hline Basal diet + Carob fiber & $49.87 \mathrm{fg} \pm 4.25$ & $2.29 \mathrm{~d} \pm 0.08$ & $0.18 \mathrm{ji} \pm 0.06$ \\
\hline Basal diet + Carob water extract & $45.44 \mathrm{gh} \pm 3.60$ & $2.23 \mathrm{ef} \pm 0.07$ & $0.23 \mathrm{eg} \pm 0.1$ \\
\hline Diabetic control group + Basal diet & $80.61 \mathrm{a} \pm 6.11$ & $4.64 a b \pm 0.39$ & $0.37 b \pm 0.12$ \\
\hline Diabetic + Basal diet + Carob fiber & $72.01 b \pm 3.20$ & $3.01 \mathrm{c} \pm 0.14$ & $0.19 \mathrm{fh} \pm 0.04$ \\
\hline Diabetic + Basal diet + carob water extract & $69.00 \mathrm{bc} \pm 2.82$ & $3.20 \mathrm{~d} \pm 0.29$ & $0.21 \mathrm{fg} \pm 0.09$ \\
\hline Hypercholesterolemia control group & $83.63 a \pm 5.50$ & $5.05 a \pm 0.23$ & $0.52 \mathrm{a} \pm 0.18$ \\
\hline $\begin{array}{l}\text { Hypercholesterolemia + Basal diet + Carob } \\
\text { fiber }\end{array}$ & $60.68 \mathrm{de} \pm 2.21$ & $4.64 a b \pm 0.36$ & $0.32 b c \pm 0.08$ \\
\hline $\begin{array}{l}\text { Hypercholesterolemia + Basal diet + carob } \\
\text { water extract }\end{array}$ & $63.06 \mathrm{~cd} \pm 1.63$ & $4.75 \mathrm{ab} \pm 0.20$ & $0.23 \mathrm{dg} \pm 0.08$ \\
\hline
\end{tabular}

In diabetic and hypercholesterolemia groups there were significant increment in urea, uric acid and creatinine comparing to control (basal diet). Administration of carob fiber and water extract induced significant decrement in urea, uric acid and creatinine in diabetic group and significant decrement in urea and creatinine in hypercholesterolemia groups comparing with control groups. The obtained results are in agreement with those reported by Mahgoub (2010), Ali et al. (2012) and Shalby et al. (2012). The effect of carob fiber and water extract on hematological parameters of the rats are shown in Table (9). Administration of carob fiber and water extract induced significant increment in WBC in diabetic groups. In hypercholesterolemia group administrated with carob fiber showed significant increment in $\mathrm{RBC}$ count while, carob water extract showed increment in RBC count and decrement in WBC count. Gulay et al. (2012) reported that hematological parameters showed no significant differences between control and treated animals with carob bean extract.

Table 9. Effect of orally intake carob fiber and water extract of carob on hematological parameters.

\begin{tabular}{llll}
\hline Groups & $\mathrm{RBC}$ & $\mathrm{WBC}$ & $\mathrm{Hb}$ \\
& $\left(\mathrm{X} 10^{6} / \mu \mathrm{l}\right)$ & $\left(\mathrm{X} 10^{3} / \mu \mathrm{dl}\right)$ & $14.34 \mathrm{ac}$ \\
& $6.7 \mathrm{ab}$ & $15.78 \mathrm{eg}$ & \pm 0.61 \\
\hline Control Basal diet & \pm 0.31 & \pm 2.39 & $13.98 \mathrm{bd}$ \\
& $7.03 \mathrm{ab}$ & $15.70 \mathrm{fg}$ & \pm 0.95 \\
\hline Basal diet + Carob fiber & \pm 0.30 & \pm 0.70 & $15.1 \mathrm{a}$ \\
& $7.49 \mathrm{a}$ & $17.06 \mathrm{de}$ & \pm 0.67 \\
\hline Basal diet + Carob water extract & \pm 0.14 & \pm 1.95 & $13.34 \mathrm{df}$ \\
& $6.54 \mathrm{ac}$ & $14.6 \mathrm{~g}$ & \pm 1.36 \\
\hline Diabetic control group + Basal diet & \pm 0.39 & \pm 2.41 & $12.66 \mathrm{f}$ \\
& $6.33 \mathrm{bc}$ & $16.34 \mathrm{df}$ & \pm 0.31 \\
\hline Diabetic + Basal diet + Carob fiber & \pm 0.22 & \pm 3.20 & $14.02 \mathrm{bd}$ \\
& $7.03 \mathrm{ab}$ & $19.16 \mathrm{~b}$ & \pm 0.72 \\
\hline Diabetic + Basal diet + carob water extract & \pm 0.23 & \pm 3.6 & $12.86 \mathrm{ef}$ \\
& $5.76 \mathrm{c}$ & $17.60 \mathrm{~cd}$ & \pm 0.06 \\
\hline Hypercholesterolemia control group & \pm 0.55 & \pm 1.64 & $13.58 \mathrm{cf}$ \\
& $7.07 \mathrm{ab}$ & $18.85 \mathrm{bc}$ & \pm 0.12 \\
\hline Hypercholesterolemia + Basal diet + Carob fiber & \pm 0.06 & \pm 1.82 & $13.8 \mathrm{be}$ \\
\end{tabular}

$a, b, c, \ldots f$ means within column with different letters differ significantly $(\mathrm{p} \leq 0.05)$ from each other means followed by the same letter don't differ at 0.05 probability level.

Each value represents the mean of 5 rats \pm S.E 


\section{References}

A. O. A. C.(2005)."Offical Methods of Analytical"18th ED.Assoiation of Ofical Analytical Chemists, Gaithersburg, Maryland, U.S.A.

Abdel-Rahim, E.A. El-Beltagi H.S., and Romela R.M. (2013). White Bean seeds and Pomegranate peel and fruit seeds as hypercholesterolemic and hypolipidemic agents in albino rats. Grasasy Aceites, 64 (1):50-58.

Abd Razik , B.M., Hasan , H.A., and Murtadha, M. K. (2012) . The Study of Antibacterial Activity of Plantago Major and Ceratonia Siliqua . The Iraqi postgraduate medical journal.11,(1), 130- 135.

Abugri, D. A and McElhenney, W.H. (2013). Extraction of total phenolic and flavonoids from edible wild and cultivated medicinal mushrooms as affected by different solvents. J. Nat. Prod. Plant Resour., 3 (3):37-42.

Aissani , N., Coroneo, V., Fattouch, S., and Caboni, P. (2012). Inhibitory effect of carob (ceratonia siliqua) leaves methanolic extract on listeria monocytogenes. Journal of Agricultural and Food Chemistry 10;60(40):9954-9958.

Allain, C.C.; Poon, L.S.; Chan, C.S. and Richmond, W. (1974). Enzymatic determination of total serum cholesterol. Clin. Chem., 20: 470475.

Ali, M. M. E., Abd El-Megeid, A.A. and Mostafa, R. A. A. (2012). Effect of some levels from ginseng, barley and carob on lipid profile and kidney functions of rats fed on high fructose diets. Journal of American Science.8(10): 152162.

Al-Rawahi, A. S.; Rahman, M. S.; Guizani, N. and Essa, M, M. (2013). Chemical Composition, Water Sorption Isotherm, and Phenolic Contents in Fresh and Dried Pomegranate Peels. Drying Technology, 31: 257-263.

Avallone, R, Plessi, M, Baraldi, M, Monzani, A. (1997). Determination of chemical composition of carob (Ceratonia siliqua): Protein, fat,

carbohydrates and tannins. J. Food Compost. Anal. 10:166-172.

Benchikh, Y and Louailèche, H.(2014). Effects of extraction conditions on the recovery of phenolic compounds and in vitro antioxidant activity of carob (Ceratonia siliqua L.) pulp. Acta Botanica Gallica: Botany Letters Volume 161, ( 2): 12531260.

Buko, V., Luklvskaya, O., Nkitin, V., Tarasov, Y., Zavodink, L., Borodassky, A., Cioren, T., Shetein, B., Tanz, B., Mudermann, K.J (1996). Hepatic and pancreatic effects of polyenoyl phatidyl choline in rats with alloxan induce diabetes. Cell Biochem. Furnet. 14(2):131-137.
Çam, M. and Hışıl, Y. (2010). Pressurised water extraction of polyphenols from pomegranate peels. Food Chem. 123(3):878-885

Custodio.L., Carneiro,M.F ., and Romano.A., (2005). Microsporogenesis and anther culture in carob tree (Ceratonia siliqua L). Scince Horticulturae, 104: 65-77.

Djeridane, A., Yousfi, M., Nadjemi, B., Boutassouna, D., Stocker, P., Vidal, N., (2006). Antioxidant activity of some Algerian medicinal plants extracts containing phenolic compounds. Food Chem. 97(4):654-660.

Dubois, M.; Smith, F.; Gilles, K.A.; Hammilton, J.K. and Robers, P.A. (1956): Colorimetric method to determination of sugars and related substances. Anal. Chem. 28 (3): 350-356.

Elfalleh, W.; Nasri, N.; Marzougui, N.; Thabti, I.; M'rabet, A.; Yahya, Y.; Lachiheb, B.; Guasmi, F. and Ferchichi, A. (2009). Physico-chemical properties and DPPH-ABTS scavenging activity of some local pomegranate (Punica granatum) ecotypes. Int. J. Food Sci. Nutr. 60(2):197-210.

Farrugia, G., Balzan R (2012). Oxidative stress and programmed cell

death in yeast. Front. Oncol. 2:1-21.

Forestieri, A. M., Galati, E. M., Trovato, A. and Tumino, G. (2006). Effects of guar and carob gums on glucose, insulin and cholesterol plasma levels in the rat. Phytotherapy research. 3(1): 1-4.

Fossati, P. and Principe, L. (1982). Enzymatic colorimetric method to determination triglycerides. Clin. Chem., 28: 2077.

Gulay, M. S, Yildiz -Gulay,O.,Ata,A.,Balic,A and Demirta,S. (2012) . Toxicological evaluation of carob ( Ceratonia silique) bean extracts in male Zealand white rabbits . Journal of Analatical and Veterinary Advances. 11(11):1853-1857.

Hsouna, A. B, ALayed, A. S and Abdallah , E M.(2012). Evaluation of antimicrobial activities of crude methanolic extract of pods of Ceratonia siliqua L. against some pathogens and spoilage bacteria. African Journal of Microbiology Research 6(14), 3480-3484.

Hussein , A. M.S ., Shedeed, N Am,,. Abdel-Kalek, H. H., and Shams El-Din, M.H.A.(2011). Antioxidative, antibacterial and antifungal activities of tea infusions from berry leaves, carob and doum . Pol. J. Food Nutr. Sci. 61(3): 201-209.

Iddamaldeniya, S.S., Thaberw,M.I., Wickramasingghel, S.M.D., Ratnatunge, N. N. and Thammitiyagodage, M.G., (2006). A longterm investigation of the anti-hepatocarcinogenic potential of an indigenous medicine comprised of niglla sativa, Hemidesmus indicine and Smilax glabra. J. of Carcinogenesis5:11-24.

Kivcak, B., Mert, T. and Ztcrk, H. T. (2002) . Antimicrobial and Cytotoxic activities of 
Ceratonia siliqua L. extracts . Turk J Biol 26, 197-200.

Khlifa , M. , Bahloul, A., Kitane, S.(2013). Determination of Chemical Composition of Carob Pod (Ceratonia Siliqua L) and its Morphological Study. J. Mater. Environ. Sci. 4 (3): 348-353.

Lobo V, Patil A, Phatak A, Chandra N (2010). Free radicals, antioxidants and functional foods: Impact on human health. Pharmacogn. Rev. 4:118-126.

Mahgoub, M. A. (2010): Biochemical Studies on Nephroprotective Effect of Carob (Ceratonia siliqua L.) Growing in Egypt. Nature and Science.8(3):41-47.

Mokhtari , M, Sharifi, S., and Shahamir T. M.(2011). The Effect of Hydro - Alcoholic Seeds Extract of Ceratonia siliqua on the Blood

Glucose and Lipids Concentration in Diabetic Male Rats. International Conference on Life Science and Technology 3:82-87.

Orak, H.H.; Demirci, A.Ş. and Gümüş , T. (2011). Antibacterial and antifungal activity of pomegranate (Punica granatum cv.) peel. Electronic Journal of Environmental Agricultural and Food Chemistry 10(3): 1958-1969.

Okonogi, S., Duangrat, C., Anuchpreeda, S., tachakittirungrod, S., Chowwanapoonpohn, S. (2007). Comparison of antioxidant capacities and cytotoxicities of certain fruit peels. Food Chem. 103(3):839-846

Reeves, P.G; Nilsen,F.H. and Fahey, G.C.(1993):AIN-93purified diets for laboratory rodents: Final report of the American Institute of Nutrition ad hoc writing committee on the reformulation of the AIN-76Arodent diet. J.Nut., 123:1939-1951.

Roso , B, . Quintela, R,- J, C, de la Fuente, E, Haya, J, and Olleros, L, P. (2010) . Insoluble Carob Fiber Rich in Polyphenols Lowers Total and LDL Cholesterol in Hypercholesterolemic Sujects . Plant Foods for Human Nutrition ;65(1):50-60.

SAS. (1996). SAS procedure Guide. (Version 6.12Ed). Institute Inc., Cary,NC, USA.

Sebai, H., Souli, A., Chehimi,L., Rtibi, K., Amri,M., El-Benna.,M and Sakly,M.,(2013).In vitro and in vivo antioxidant properties of Tunisian carob (Ceratonia siliqua L.). Journal of Medicinal Plants Research. 7(2): 85-90.

Shalby, A.B., Hamza, A.H. and Ahmed, H.H.(2012). New insights on the antiinflammatory effect of some Egyptian plants against renal

dysfunction induced by cyclosporine. European Review for Medical and Pharmacological Sciences 16: 455-461.

Tabatabai, A .,and Li S. (2000). Dietary fiber and type 2 diabetes. Clin Excell Nurse Pract.; 4(5):272-2766.
Tietz, N.W,(1976a). Fundamentals of Clinical Chemistery W.B.Saunders co.,Philadelphia.p. 243.

Tietz, N.W,(1976b). Fundamentals of Clinical Chemistery W.B.Saunders co.,Philadelphia.p.999-1002.

Tietz., N,W(1986). Textbook of clinical chemistry. WB saunders, Philadelphia, pp1271-1281.

Tietz, N.W,(1990). Clincal guide to laboratory tests. 2 nd ED. philadelphia: WB Saunders.566.

Trinder, P. (1969). Determination of Glucose in blood using glucose-oxidase with an alternative oxygen acceptor. Ann. Clin. Biochem. 6: 24-27.

Vassault, A.; Grafmeyer, D.; Naudin, C.; Dumont, G.; Bailly, M. and Henny J. (1986). Protocole de validation de techniques. Ann. Biol. Clin. 44: 686-745.

Walters, M.I. and Gerarde, H.W. (1970). An ultramicro method for the determination of conjugated and total bilirubin in serum or plasma. Microchemical Journal 15(2): 231-243.

Wursch, $P$.(1979) Influence of Tannin-Rich Carob Pod Fiber on the Cholesterol Metabolism in the Rat. J. Nutr. 109: 685-692.

Yaser, A. J .,Muneer,A, Abdelhafid,B., Fawzia, A.(2014). Chemical Composition, Phytochemical Constituents and Antioxidant Activities of the Seeds Extract of Apiumgraveoleus L from Yemen. Research Journal of Pharmaceutical, Biological and Chemical Sciences.5(1):10741087.

Yim, H.S., ,Chye, F,Y., Ho, S.K. and Ho, C.W.(2009). Phenolic profiles of selected edible wild mushrooms as affected by extraction solvent, time and temperature. As. J. Food AgInd, 2(03): 392-401.

Young, D.S.; Thomas, D.W.; Friedman, R.B. and Pestaner, L.C. (1972). Effects of drugs on clinical laboratory tests. Clinical Chemistry 18(1) 1041-1303.

Young, D.S.; Pestaner, L.C. and Gibberman, V. (1975). Effect of drugs on clinical laboratory tests. Clinical Chemistry, 21:1D-432D.

Zam, W., Bashour, G., Abdelwahed, W., and Khayata, W., (2013) . Simple and fast method for the extraction of polyphenol and the separation of proanthocyanidins from carob pods. Sch. Acad. J. Pharm., 2(5):375-380.

Zunft, H.J., Luder, W, Harde, A., Haber, B., Graubaum, H.J.and Gruenwald, J. (2001). Carob pulp preparation for treatment of hypercholesterolemia. Adv. Ther. 18:230-236.

Zunft, H.J., Luder, W., Harde, A., Haber, B., Graubaum, H.J, Koebnick C, and Grunwald, J. (2003). Carob pulp preparation rich in insoluble fibre lowers total and LDL cholesterol in hypercholesterolemia patients.

Eur. J. Nutr. 42:235-242. 


\section{الملخص}

تهذف هذه الدراسة إلي دراسة أفضل طريقه لاستخلاص البولي فينول و الثانينات والفلافونويدات ومضادات الأكسدة من الخروب و قد تم استخدام

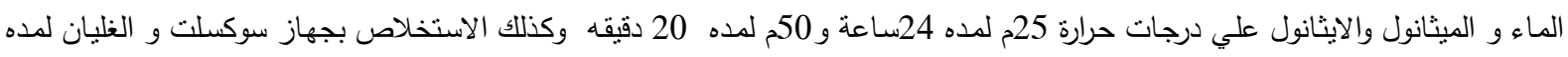

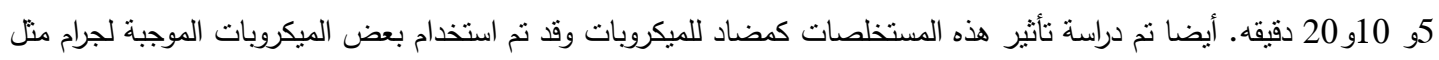

( Staphylococcus aureus, Listeria monocytogenese, Bacillus megaterium and Bacillus cereus)

$$
\text { والسالبة لجرام مثل }
$$

( Escherichia coli, Klebsiella pneumonia and Salmonella typi)

والفطريات مثل (Aspergillus niger and Candida albicans ) كما تم دراسة تأثير الياف الخروب والمستخلص المائي للخروب علي

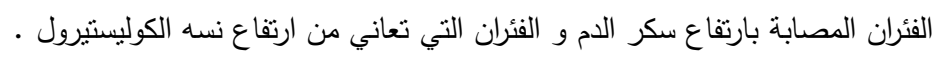

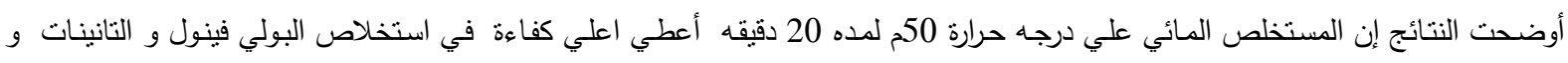
الفلافونويدات بينما مستخلص الميثانول علي 50م لمده 20 دقيقه أوضح اعلي كفاءة في استخلاص مضادات الأليات الأكسدة.

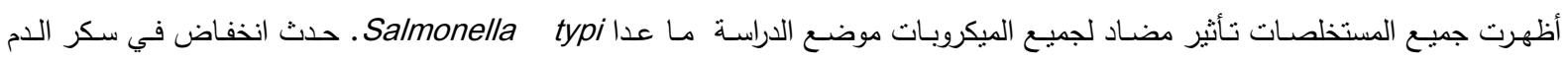

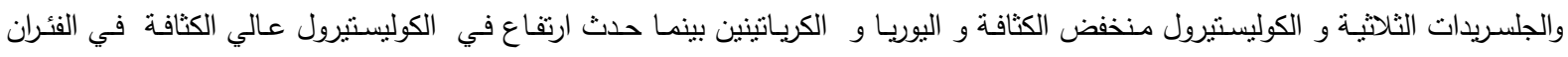

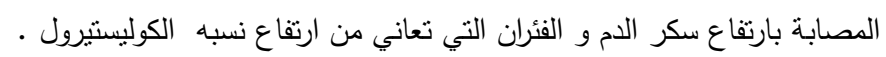

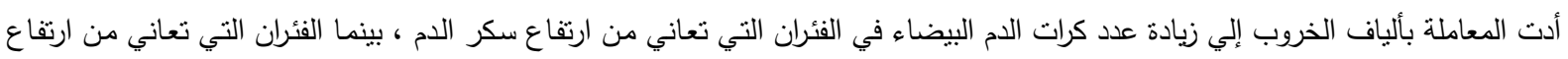

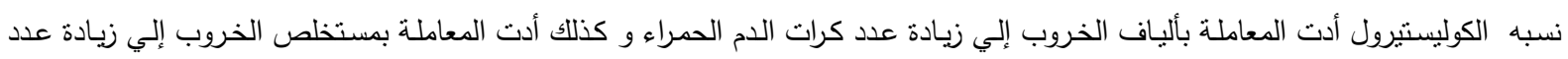

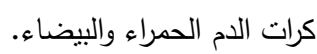

